\title{
Implementing Deutsch-Jozsa algorithm using light shifts and atomic ensembles
}

\author{
Shubhrangshu Dasgupta, Asoka Biswas, and G. S. Agarwal* \\ Physical Research Laboratory, Navrangpura, Ahmedabad 380 009, India
}

(Received 6 April 2004; published 27 January 2005)

\begin{abstract}
We present an optical scheme to implement the Deutsch-Jozsa algorithm using ac Stark shifts. The scheme uses an atomic ensemble consisting of four-level atoms interacting dispersively with a field. This leads to a Hamiltonian in the atom-field basis which is quite suitable for quantum computation. We show how one can implement the algorithm by performing proper one- and two-qubit operations. We emphasize that in our model the decoherence is expected to be minimal due to our usage of atomic ground states and freely propagating photon.
\end{abstract}

DOI: 10.1103/PhysRevA.71.012333

PACS number(s): 03.67.Lx, 03.65.-w, 42.50.-p

\section{INTRODUCTION}

Quantum computers are expected to be much faster than the classical ones, especially in performing some specific jobs, like factorization, searching, etc. For example, any function of $N$ binary variables (bits) can be identified as a constant (single valued) or balanced (bivalued) by a single enquiry through the well-known Deutsch-Jozsa algorithm [1], whereas classically it needs up to $\left(2^{N-1}+1\right)$ enquiries [2]. Let us consider $N=2$ bits. There are $2^{N}=4$ possible states 00 , $01,10,11$, which we can designate as four values of a variable $x=0,1,2,3$. One requires $2^{N-1}+1=3$ evaluations of the function $f(x)$ to determine its characteristic, whereas this algorithm needs only one evaluation for the same. Further, in Grover's search algorithm [3], searching for a specific state from an unsorted database of $N$ states requires $O(\sqrt{N})$ repetitions of a certain unitary operation, whereas any classical computer would need $O(N)$ attempts. All these faster algorithms have been made possible by the special property of a quantum state, which enables one to apply the same unitary operations simultaneously on a number of basis states (quantum parallelism).

These algorithms have been implemented using nuclear magnetic resonance technologies, trapped ions, etc. Bulk nuclear magnetic resonance has been used to implement scalable versions of the Deutsch-Jozsa algorithm [4] and Grover's algorithm [5]. The Deutsch-Jozsa algorithm using selective pulses [6] and quadrupolar nuclei [7] in nuclear magnetic resonance systems has been demonstrated. Guide et al. [8] have implemented the Deutsch-Jozsa algorithm in an ion trap system. Theoretical proposals to implement Grover's algorithm using trapped ions [9] and cavity quantum electrodynamics [10] have been reported.

In this paper, we propose an optical system to implement the Deutsch-Jozsa algorithm. We use an ensemble of fourlevel atoms interacting dispersively with a field to obtain an effective Hamiltonian like the one on which a typical nuclear magnetic resonance experiment is based [11]. This enables us to implement various two-qubit and single-qubit opera-

\footnotetext{
*Present address: Department of Physics, Oklahoma State Univer-
} sity, Stillwater, OK 74078, USA. tions to realize the Deutsch-Jozsa algorithm. The entire algorithm can be implemented by using, for example, the clock transitions in the $\mathrm{Cs}$ atomic ensemble.

The structure of the paper is as follows. In Sec. II, we briefly discuss the basic method to implement the DeutschJozsa algorithm. In Sec. III, we describe the model and the relevant Hamiltonian. In Sec. IV, we provide the required pulse sequence to implement the algorithm using our model.

\section{BASIC REQUIREMENTS OF THE DEUTSCH-JOZSA ALGORITHM}

To start with, let us recall the main features of this algorithm. This algorithm is used to determine whether a given function is constant or balanced. In the simplest case, we consider a one-bit output function $f(x)$ (which can take only the value 0 or 1$)$ of a one-bit input $x(x=0$ or 1$)$. If $f_{1}(x)$ $=0$ and $f_{2}(x)=1$, then these functions are constant. On the other hand, if $f_{3}(x)=x$ and $f_{4}(x)=$ NOT $x$, then they are balanced functions. In Table I, we show the above functions $f_{n}(x)$. The one-bit version of the Deutsch-Jozsa algorithm $(N=1)[8]$ determines the characteristics of the function by a single function call, contrary to its classical counterpart, which requires $2^{N-1}+1=2$ function calls to check whether the function is constant or balanced. In fact, in this algorithm, one calculates the value $f(0) \oplus f(1)$ (where $\oplus$ denotes addition modulo 2). This yields 0 (or 1 ) for a constant (or balanced) function.

In Fig. 1, we show the basic circuit [2] to perform one-bit version of the Deutsch-Jozsa algorithm. We start with the initial state of the two qubits as

TABLE I. Different one-bit functions showing their characterizations.

\begin{tabular}{ccccc}
\hline \hline \multirow{2}{*}{ Input } & \multicolumn{2}{c}{ Constant } & \multicolumn{2}{c}{ Balanced } \\
$x$ & $f_{1}(x)$ & $f_{2}(x)$ & $f_{3}(x)$ & $f_{4}(x)$ \\
\hline 0 & 0 & 1 & 0 & 1 \\
1 & 0 & 1 & 1 & 0 \\
\hline \hline
\end{tabular}




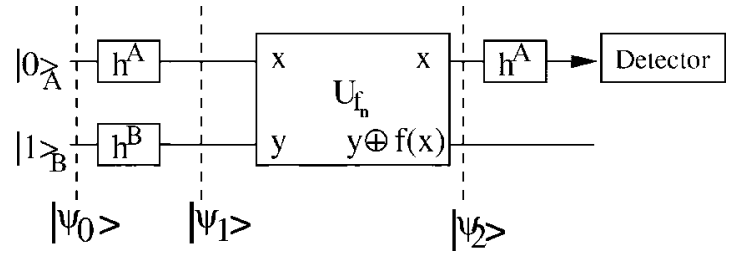

FIG. 1. Basic circuit to perform the Deutsch-Jozsa algorithm. The detector measures the state $|0\rangle$ or $|1 \mathrm{f}\rangle$ of the qubit $A$.

$$
\left|\psi_{0}\right\rangle=|0\rangle_{A}|1\rangle_{B} .
$$

The transformations $h^{A}$ and $h^{B}$ create superpositions $(|0\rangle$ $+|1\rangle)_{A} / \sqrt{2}$ and $(|0\rangle-|1\rangle)_{B} / \sqrt{2}$ from the input states $|0\rangle_{A}$ and $|1\rangle_{B}$, i.e.,

$$
\left|\psi_{1}\right\rangle=\frac{1}{\sqrt{2}}(|0\rangle+|1\rangle)_{A} \frac{1}{\sqrt{2}}(|0\rangle-|1\rangle)_{B} .
$$

In Fig. 1, $U_{f_{n}}$ is the unitary operator corresponding to each function $f_{n}(x)(n \in 1, \ldots, 4)$ shown in Table I. This two-bit operator yields the result $|x\rangle_{A}|y\rangle_{B} \rightarrow|x\rangle_{A}\left|y \oplus f_{n}(x)\right\rangle_{B}$, where $x, y \in 0,1$. One can identify different $U_{f_{n}}$ 's as follows: $U_{f_{1}}$ is the identity operation and $U_{f_{2}}$ is the NOT operation on qubit $B ; U_{f_{3}}$ is the controlled-NOT (CNOT) operation and $U_{f_{4}}$ is the zero-CNOT operation in the two-qubit basis $\left(\left|0_{A}, 0_{B}\right\rangle,\left|0_{A}, 1_{B}\right\rangle,\left|1_{A}, 0_{B}\right\rangle,\left|1_{A}, 1_{B}\right\rangle\right)$ [8].

The output $\left|\psi_{2}\right\rangle$ after the operation $U_{f n}$ on the state $\left|\psi_{1}\right\rangle$ becomes

$$
\begin{aligned}
\left|\psi_{2}\right\rangle= & \frac{1}{\sqrt{2}}\left[|0\rangle_{A}\left|0 \oplus f_{n}(0)\right\rangle_{B}-|0\rangle_{A}\left|1 \oplus f_{n}(0)\right\rangle_{B}\right. \\
& \left.+|1\rangle_{A}\left|0 \oplus f_{n}(1)\right\rangle_{B}-|1\rangle_{A}\left|1 \oplus f_{n}(1)\right\rangle_{B}\right] .
\end{aligned}
$$

It can be easily verified that for any $f_{n}(x)$ as defined in Table I, the above state $\left|\psi_{2}\right\rangle$ becomes factorized in the Hilbert space of $A$ and $B$. This can be written as (ignoring the overall phase factor)

$$
\left|\psi_{2}\right\rangle \equiv \frac{1}{\sqrt{2}}(|0\rangle \pm|1\rangle)_{A} \frac{1}{\sqrt{2}}(|0\rangle-|1\rangle)_{B},
$$

where the output state of the qubit $A$ becomes a superposition with positive (negative) sign, if the function is constant (balanced). Hence, after performing the final one-qubit rotation $h^{A}$, one can detect the qubit $A$ in state $|0\rangle(|1\rangle)$ to identify that the function is constant (balanced). Thus, to perform a one-bit Deutsch-Jozsa algorithm one has to be able to construct certain two-qubit gates as well as single-qubit rotation gates.

\section{MODEL}

\section{A. Dispersive interaction between an ensemble of atoms and a single photon}

To obtain the required single-qubit and two-qubit operators so as to perform the Deutsch-Jozsa algorithm, we consider a four-level atomic configuration of energy levels as shown in Fig. 2. The dipole moment vectors between the

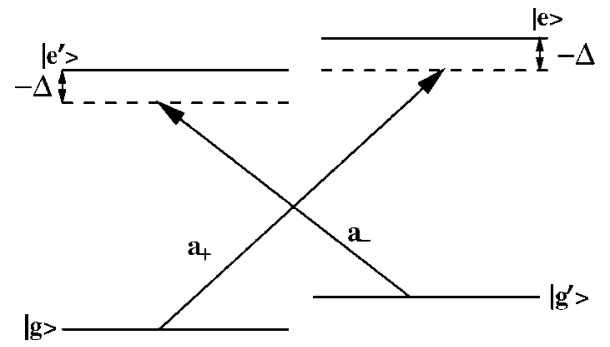

FIG. 2. Relevant level configuration for implementing the Deutsch-Jozsa algorithm. The excited levels $|e\rangle$ and $\left|e^{\prime}\right\rangle$ are coupled with the ground levels $|g\rangle$ and $\left|g^{\prime}\right\rangle$, respectively, by the orthogonal polarization modes $a_{+}$and $a_{-}$. These modes are detuned from the corresponding transitions by the same amount $-\Delta$.

levels $|e\rangle,|g\rangle$ and $\left|e^{\prime}\right\rangle,\left|g^{\prime}\right\rangle$ are orthogonal to each other. We note that this kind of configuration can be found in the optical domain in many atoms such as ${ }^{133} \mathrm{Cs}$ and ${ }^{199} \mathrm{Hg}$ and has been studied in the context of spin squeezing and quantum nondemolition measurements [12], for generating mesoscopic superposition states in atomic gases [13], and in quantum communication between two different atomic ensembles [14]. Two trapped atoms with such configurations interacting with $\sigma$-polarized laser fields have been shown to perform quantum logic gates conditioned on photon detection [15].

In our model, an atomic ensemble consisting of $N$ atoms, each with relevant level configuration as shown in Fig. 2, interacts with a single photon. The states of the photon are to be labeled as $|1,0\rangle$ and $|0,1\rangle$ which refer to, for example, the two orthogonal polarization components $\sigma_{ \pm}$, respectively. These components interact with the $|e\rangle \leftrightarrow|g\rangle$ and $\left|e^{\prime}\right\rangle \leftrightarrow\left|g^{\prime}\right\rangle$ transitions, respectively. Both the components are equally detuned from the corresponding transition by an amount $-\Delta$ (see Fig. 2). Let us consider that the coupling of both the components is the same and equal to $g$. The interaction Hamiltonian of the ensemble-photon system under the rotating wave approximation can be written as

$$
\begin{aligned}
H= & -\hbar \Delta \sum_{j=1}^{N}\left(\left|e_{j}\right\rangle\left\langle e_{j}|+| e_{j}^{\prime}\right\rangle\left\langle e_{j}^{\prime}\right|\right)-i \hbar g \sum_{j=1}^{N}\left[\left|e_{j}\right\rangle\left\langle g_{j}|| 0,0\right\rangle\langle 1,0|\right. \\
& \left.+\left|e_{j}^{\prime}\right\rangle\left\langle g_{j}^{\prime}|| 0,0\right\rangle\langle 0,1|-\text { H.c. }\right] .
\end{aligned}
$$

Now in the large detuning regime $(\Delta \gg g)$, the ground levels $|g\rangle$ and $\left|g^{\prime}\right\rangle$ of each atom in the ensemble get Stark shifted. The energy shift of the level $|g\rangle$ of each atom can be calculated using second-order perturbation theory as follows:

$$
\frac{|\langle g, 1,0|H| e, 0,0\rangle|^{2}}{E_{g}-E_{e}}=\frac{\hbar|g|^{2}}{\Delta}=\hbar \lambda,
$$

where $\lambda=|g|^{2} / \Delta$. Here $E_{k}=\langle k|H| k\rangle(k=e, g)$ are the energies of the corresponding levels. Note that the level $|g\rangle$ gets Stark shifted only if there is a photon in the $a_{+}$mode. In a similar way, the level $\left|g^{\prime}\right\rangle$ also gets Stark shifted by the same amount, if there is a photon in the mode $a_{-}$. Note that the levels $|e\rangle$ and $\left|e^{\prime}\right\rangle$ will not be populated under the action of the Hamiltonian (5) as we are working in the large detuning regime. On the basis of Stark shifts, the effective Hamiltonian for each atom $j$ can be written as 


$$
H_{\text {eff }}^{j}=\hbar \lambda\left[\left|g_{j}, 1,0\right\rangle\left\langle g_{j}, 1,0|+| g_{j}^{\prime}, 0,1\right\rangle\left\langle g_{j}^{\prime}, 0,1\right|\right] .
$$

The effective Hamiltonian of the ( $N$-atoms $)+($ photon $)$ system becomes

$$
\begin{aligned}
H_{\text {eff }} & =\sum_{j=1}^{N} H_{\text {eff }}^{j} \\
& =\hbar \lambda \sum_{j=1}^{N}\left[\left|g_{j}\right\rangle\left\langle g_{j}|| 1,0\right\rangle\left\langle 1,0|+| g_{j}^{\prime}\right\rangle\left\langle g_{j}^{\prime}|| 0,1\right\rangle\langle 0,1|\right] \\
& =-2 \hbar \lambda\left[\hat{S}^{z} \hat{R}^{z}-\frac{1}{4} \hat{N} \hat{\mathbf{1}}\right],
\end{aligned}
$$

where

$$
\begin{gathered}
\hat{S}^{z}=\frac{1}{2} \sum_{j=1}^{N}\left(\left|g_{j}^{\prime}\right\rangle\left\langle g_{j}^{\prime}|-| g_{j}\right\rangle\left\langle g_{j}\right|\right), \\
\hat{R}^{z}=\frac{1}{2}(|1,0\rangle\langle 1,0|-| 0,1\rangle\langle 0,1|), \\
\hat{N}=\sum_{j=1}^{N}\left(\left|g_{j}^{\prime}\right\rangle\left\langle g_{j}^{\prime}|+| g_{j}\right\rangle\left\langle g_{j}\right|\right), \\
\hat{\mathbf{1}}=(|1,0\rangle\langle 1,0|+| 0,1\rangle\langle 0,1|) .
\end{gathered}
$$

In Appendix A, we provide a rigorous derivation of the effective Hamiltonian (8). Note that the Hamiltonian (8c) has a form that is similar to those used for quantum computation with nuclear magnetic resonance systems [11]. Hence it is possible to perform all the required logic gate operations with the atoms and the photon in the present model as are possible with the standard nuclear magnetic resonance Hamiltonian.

\section{B. Two-qubit and single-qubit operations}

The system of $N$ atoms has $2^{N}$ states. We identify two states $\Pi_{j=1}^{N}\left|g_{j}\right\rangle\left(\equiv|0\rangle_{A}\right)$ and $\Pi_{j=1}^{N}\left|g_{j}^{\prime}\right\rangle\left(\equiv|1\rangle_{A}\right)$ to identify one qubit. The other qubit will consist of two polarization states of the photon $|0,1\rangle\left(\equiv|0\rangle_{F}\right)$ and $|1,0\rangle\left(\equiv|1\rangle_{F}\right)$. Because the relevant atomic levels are the ground levels, there is no decoherence due to the spontaneous emission of the atom. Further, we are considering a photon in free space which can have a long decay time.

\section{Two-qubit operation}

We can write the unitary operator $U_{\text {eff }}=\exp \left[-i H_{\text {eff }} t / \hbar\right]$ corresponding to the effective Hamiltonian (8). Under the action of this operator, the state $|0\rangle_{F}|1\rangle_{A}$ evolves as follows:

$$
\begin{aligned}
U_{\mathrm{eff}}|0\rangle_{F}|1\rangle_{A} & =e^{-i H_{\mathrm{eff}} t / \hbar} \prod_{j=1}^{N}\left|g_{j}^{\prime}\right\rangle|0,1\rangle=e^{-i t \Sigma_{j=1}^{N} \lambda} \prod_{j=1}^{N}\left|g_{j}^{\prime}\right\rangle|0,1\rangle \\
& =e^{-i \lambda N t} \prod_{j=1}^{N}\left|g_{j}^{\prime}\right\rangle|0,1\rangle .
\end{aligned}
$$

The state $|1\rangle_{F}|0\rangle_{A}$ will evolve in a similar way as above and acquires a phase $e^{-i \lambda N t}$. However, the states $|0\rangle_{F}|0\rangle_{A}$ and $|1\rangle_{F}|1\rangle_{A}$ will not evolve under the action of $U_{\text {eff }}$. Thus, for $\lambda N T=\pi / 2$, the basis states of the two qubits evolve as follows:

$$
\begin{gathered}
|0\rangle_{F}|0\rangle_{A} \rightarrow|0\rangle_{F}|0\rangle_{A}, \\
|0\rangle_{F}|1\rangle_{A} \rightarrow-i|0\rangle_{F}|1\rangle_{A},
\end{gathered}
$$

$$
\begin{gathered}
|1\rangle_{F}|0\rangle_{A} \rightarrow-i|1\rangle_{F}|0\rangle_{A}, \\
|1\rangle_{F}|1\rangle_{A} \rightarrow|1\rangle_{F}|1\rangle_{A},
\end{gathered}
$$

where $T$ is the interaction time of the atomic ensemble with the photon.

We next show how this realization of two-qubit gate is feasible. We use some of the examples considered previously $[12,16,17]$. We consider the clock transition in ${ }^{133} \mathrm{Cs}$ with atomic frequency $2 \pi \times 3.517 \times 10^{14} \mathrm{~s}^{-1}$ and dipole moment element $3.797 \times 10^{-29} \mathrm{C} \mathrm{m}$. For a Cs atomic cloud [12] of length $5 \mathrm{~mm}$ and cross section $\sim 0.1 \mathrm{~mm}^{2}$ (volume of the cloud $=5 \times 10^{-10} \mathrm{~m}^{-3}$ ), the atom-photon coupling constant $g$ becomes $1.84 \times 10^{6} \mathrm{~s}^{-1}$. The number of atoms $N$ being $\sim 10^{8}$ (for an atomic density $\sim 3 \times 10^{11} \mathrm{~cm}^{-3}$ ), we can calculate the required detuning $\Delta=3.59 \mathrm{GHz}=1951 \mathrm{~g}$ so as to satisfy the condition $\lambda N T=\pi / 2$. If we choose [16] a volume 3 $\times 10^{-9} \mathrm{~m}^{-3}$, the atom-photon coupling constant $\mathrm{g}$ becomes $7.54 \times 10^{5} \mathrm{~s}^{-1}$. Thus for $N=2 \times 10^{8}$ and $T=10^{-11} \mathrm{~s}$ (corresponding to a cloud of length $3 \mathrm{~mm}$ ), we get $\Delta$ $=0.724 \mathrm{GHz}=960 \mathrm{~g}$. Note that in both the examples, the approximation $\Delta \gg g$ is satisfied.

Since the result (11) is the key to the successful implementation of the Deutsch-Jozsa algorithm, we have done numerical simulations using the full Hamiltonian (5) to verify the validity of the result (11). Let us denote by $c_{0}$ the amplitude of the state $\left|1_{F}\right\rangle\left|0_{A}\right\rangle$. Then using Eqs. (B2), we obtain the exact time evolution of the amplitude $c_{0}$. In Fig. 3(a), we show the numerical solutions of Eqs. (B2) for a large detuning $\Delta=40 \mathrm{~g}$ and the initial condition $c_{0}(t=0)=1$. It is clear that at time $g T=(\Delta / g)(\pi / 2 N)=12.56$ (for $N=5)$, the imaginary part of $c_{0}$ becomes -1 , i.e., the amplitude of the state $\left|1_{F}\right\rangle\left|0_{A}\right\rangle$ becomes $-i$, verifying the two-qubit operation (11c). We also found the analytical solution for $c_{0}$ [see Eq. (B3)] which shows similar temporal behavior as in Fig. 3(a). Further, in Fig. 3(b), we show the variation of $c_{0}$ calculated at times $g T$ with respect to $\Delta$. Clearly, the imaginary part of $c_{0}$ becomes -1 only for large $\Delta$. Thus the adiabatic approximation considered in the present paper is verified. 


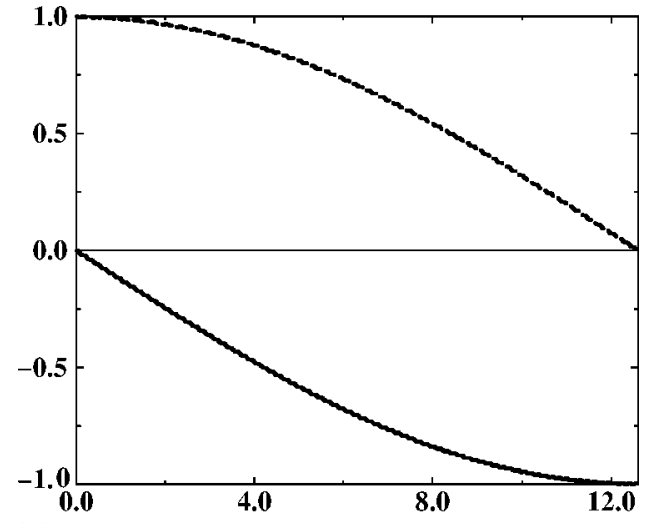

(a)

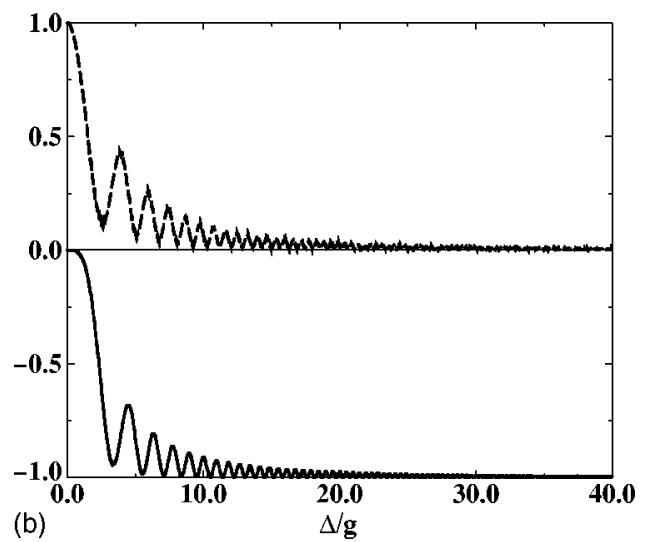

FIG. 3. Variation of the real (long-dashed line) and imaginary (solid line) parts of the coefficient $c_{0}$ (a) with time for $\Delta=40 \mathrm{~g}$ and (b) with the detuning $\Delta$. In the case of (b), $c_{0}$ is calculated at times defined by $\lambda N T=\pi / 2$. The other parameter used here is $N=5$.

\section{One-qubit operations}

Let us now introduce one-qubit operators for each atom in the ensemble and the photon, which will be used in later sections:

$$
\begin{gathered}
h_{1}=\frac{1}{\sqrt{2}}\left(\begin{array}{ll}
1 & -1 \\
1 & 1
\end{array}\right), \quad h_{2}=\frac{1}{\sqrt{2}}\left(\begin{array}{ll}
1 & i \\
i & 1
\end{array}\right), \\
h_{3}=\frac{1}{\sqrt{2}}\left(\begin{array}{ll}
1 & 1 \\
-1 & 1
\end{array}\right), \quad h_{4}=\frac{1}{\sqrt{2}}\left(\begin{array}{cc}
1 & -i \\
-i & 1
\end{array}\right) .
\end{gathered}
$$

In an atomic configuration as in Fig. 2, these inequivalent rotations can be performed by using a resonant microwave field between the ground levels $\left|g_{j}\right\rangle$ and $\left|g_{j}^{\prime}\right\rangle$ of the $j$ th atom. The relevant collective Hamiltonian for $N$ atoms can be written as

$$
H_{\mu}=-\hbar \Omega \sum_{j=1}^{N}\left[e^{i \phi}\left|g_{j}^{\prime}\right\rangle\left\langle g_{j}\right|+\text { H.c. }\right],
$$

where $\Omega$ is the coupling constant of the microwave field with the atomic ensemble and $\phi$ is the phase of the field with respect to the matrix element between the levels $\left|g_{j}\right\rangle$ and $\left|g_{j}^{\prime}\right\rangle$ of the $j$ th atom. Under the action of this Hamiltonian, the evolution of the collective states $\Pi_{j=1}^{N}\left|g_{j}\right\rangle$ and $\Pi_{j=1}^{N}\left|g_{j}^{\prime}\right\rangle$ can be expressed as

$$
\begin{aligned}
& \prod_{j=1}^{N}\left|g_{j}\right\rangle \rightarrow \prod_{j=1}^{N}\left[\cos (\Omega t)\left|g_{j}\right\rangle+i e^{i \phi} \sin (\Omega t)\left|g_{j}^{\prime}\right\rangle\right], \\
& \prod_{j=1}^{N}\left|g_{j}^{\prime}\right\rangle \rightarrow \prod_{j=1}^{N}\left[i e^{-i \phi} \sin (\Omega t)\left|g_{j}\right\rangle+\cos (\Omega t)\left|g_{j}^{\prime}\right\rangle\right] .
\end{aligned}
$$

Thus by choosing $\Omega t=\pi / 4$, one can implement the operations $h_{i}(i \in 1, \ldots, 4)$ for each atom for $\phi=-\pi / 2,0, \pi / 2$, and $\pi$, respectively. Alternatively, a combination of two resonant optical fields with $\sigma$ polarization (interacting with the $\left|e_{j}\right\rangle \leftrightarrow\left|g_{j}\right\rangle$ transition, say) and $\pi$ polarization (interacting with $\left|e_{j}\right\rangle \leftrightarrow\left|g_{j}^{\prime}\right\rangle$, say) can be used in the Raman configuration in the $j$ th atom. A suitable choice of the relative phase and amplitude of the pulses can provide the required single-qubit operations of each atom. It should be borne in mind that we do not produce a Hadamard transformation between the chosen states of the collective ensemble. However, Eq. (14) will be useful in the realization of the Deutsch-Jozsa algorithm.

The operations $h_{i}$ for the photon can be implemented using linear optical elements like absorptionless 50-50 beam splitters and phase shifters $[18,19]$. One can send the photon with either polarization through a beam splitter, so that the photon is prepared in an equal superposition state in the polarization basis after passing through it. The relative phases between the basis states at the output can be incorporated using phase shifters on the path of the photon.

We next show how the Deutsch-Jozsa algorithm can be implemented as a sequence of the above operations.

\section{IMPLEMENTING THE DEUTSCH-JOZSA ALGORITHM}

\section{A. Constructing the tools for the Deutsch-Jozsa algorithm}

Note that successful implementation of this algorithm relies on one's ability to perform all the relevant unitary operators $U_{f_{n}}$. For the present problem we choose the photon (the atomic ensemble) as the first (second) qubit.

$U_{f_{1}}$ operation. One can identify the operator $U_{f_{1}}$ as an identity operator

$$
U_{f_{1}}=\left(\begin{array}{cccc}
1 & 0 & 0 & 0 \\
0 & 1 & 0 & 0 \\
0 & 0 & 1 & 0 \\
0 & 0 & 0 & 1
\end{array}\right) .
$$

This operation on the atomic qubit does not require any interaction with the photon at all. $U_{f_{1}}$ can be written as $\mathfrak{h}_{1}^{A}\left(\mathfrak{h}_{1}^{A}\right)^{-1}$, where $\mathfrak{h}_{1}^{A}=\prod_{j=1}^{N} h_{1}^{j A}$ denotes the operation $h_{1}$ on all the atoms simultaneously, as defined in Sec. III B 2. From now onward, we denote $\mathfrak{h}=\prod_{j=1}^{N} h^{j}$.

$U_{f_{2}}$ operation. One can identify the operator $U_{f_{2}}$ as a NOT operation on the atomic qubit $\prod_{j=1}^{N}\left|g_{j}\right\rangle \leftrightarrow \Pi_{j=1}^{N}\left|g_{j}^{\prime}\right\rangle$ and it can be written in the following matrix form: 


$$
U_{f_{2}}=\left(\begin{array}{llll}
0 & 1 & 0 & 0 \\
1 & 0 & 0 & 0 \\
0 & 0 & 0 & 1 \\
0 & 0 & 1 & 0
\end{array}\right) .
$$

This operation can be achieved by applying a resonant microwave field between the levels $|g\rangle$ and $\left|g^{\prime}\right\rangle$ of each atom, such that $\Omega t=\pi / 2$ [see Eq. (14)]. Thus

$$
U_{f_{2}}=e^{-i H_{\mu} t} \text {. }
$$

Note that here $\phi$ can be chosen arbitrarily. We choose $\phi$ $=\pi / 2$ such that the NOT operation on the atomic qubit with a certain relative phase can be expressed as

$$
\prod_{j=1}^{N}\left|g_{j}\right\rangle \rightarrow e^{i N \pi} \prod_{j=1}^{N}\left|g_{j}^{\prime}\right\rangle, \quad \prod_{j=1}^{N}\left|g_{j}^{\prime}\right\rangle \rightarrow \prod_{j=1}^{N}\left|g_{j}\right\rangle .
$$

The other qubit (photon) remains redundant.

$U_{f_{3}}$ operation. To perform the $U_{f_{3}}$ operation, we choose the following operator sequence:

$$
U_{f_{3}}=\mathfrak{h}_{1}^{A} Q_{1} \mathfrak{h}_{1}^{A},
$$

where $Q_{1}$ is another two-qubit operation and $\mathfrak{h}_{1}^{A}$ represents the Hadamard rotation on the atomic qubit. The operator $Q_{1}$ is defined as

$$
Q_{1}=\mathfrak{h}_{A}^{\prime} h_{F}^{\prime} U_{\text {eff }}
$$

where $\mathfrak{h}^{\prime}=\prod_{j=1}^{N} h^{\prime j}$ and $U_{\text {eff }}=\exp \left[-i H_{\text {eff }} T / \hbar\right]$. Here, $h_{A, F}^{\prime}$ are the single-qubit operations on each atom and the photon, respectively, and can be decomposed as $h^{\prime}=h_{1} h_{4} h_{3}$.

Note that the operations $h^{\prime}$ acting on single-qubit basis states provide the following results:

$$
\begin{aligned}
h_{F}^{\prime}|0\rangle_{F}=e^{i \pi / 4}|0\rangle_{F}, & h_{F}^{\prime}|1\rangle_{F}=e^{-i \pi / 4}|1\rangle_{F}, \\
\mathfrak{h}_{A}^{\prime}|0\rangle_{A}=e^{i N \pi / 4}|0\rangle_{A}, & \mathfrak{h}_{A}^{\prime}|1\rangle_{A}=e^{-i N \pi / 4}|1\rangle_{A},
\end{aligned}
$$

Then the operator $Q_{1}$ takes the following form in the $\left(\left|0_{F} 0_{A}\right\rangle,\left|0_{F} 1_{A}\right\rangle,\left|1_{F} 0_{A}\right\rangle,\left|1_{F} 1_{A}\right\rangle\right)$ basis:

$$
Q_{1}=\operatorname{diag}\left[e^{i(N+1) \pi / 4}, e^{-i(N+1) \pi / 4},-i e^{i(N-1) \pi / 4}, e^{-i(N+1) \pi / 4}\right] .
$$

The complete operator sequence for the operator $U_{f_{3}}$ can now be written as

$$
U_{f_{3}}=\mathfrak{h}_{1}^{A}\left[\mathfrak{h}_{A}^{\prime} h_{F}^{\prime} U_{\text {eff }}\right] \mathfrak{h}_{1}^{A} .
$$

$U_{f_{4}}$ operation. We choose the following operator sequence to perform the $U_{f_{4}}$ operation:

$$
U_{f_{4}}=\mathfrak{h}_{1}^{A} Q_{2} \mathfrak{h}_{1}^{A}
$$

where $Q_{2}$ is another two-qubit operation and can be defined as

$$
Q_{2}=\mathfrak{h}_{A}^{\prime \prime} h_{F}^{\prime} U_{\text {eff }} .
$$

Here $h^{\prime}$ is defined as in Eq. (20) and $\mathfrak{h}^{\prime \prime}=\prod_{j=1}^{N} h^{\prime \prime j}$, where $h^{\prime \prime}$ $=h_{1} h_{2} h_{3}$ is another one-qubit operator. Note that the operators $h^{\prime \prime}$ result in the following:

$$
\begin{aligned}
h_{F}^{\prime \prime}|0\rangle_{F} & =e^{-i \pi / 4}|0\rangle_{F}, \quad h_{F}^{\prime \prime}|1\rangle_{F}=e^{i \pi / 4}|1\rangle_{F}, \\
\mathfrak{h}_{A}^{\prime \prime}|0\rangle_{A} & =e^{-i N \pi / 4}|0\rangle_{A}, \quad \mathfrak{h}_{A}^{\prime \prime}|1\rangle_{A}=e^{i N \pi / 4}|1\rangle_{A},
\end{aligned}
$$

Thus the operator $Q_{2}$ takes the following form in the $\left(\left|0_{F} 0_{A}\right\rangle,\left|0_{F} 1_{A}\right\rangle,\left|1_{F} 0_{A}\right\rangle,\left|1_{F} 1_{A}\right\rangle\right)$ basis:

$$
Q_{2}=\operatorname{diag}\left[e^{-i(N-1) \pi / 4}, e^{i(N-1) \pi / 4},-i e^{-i(N+1) \pi / 4}, e^{i(N-1) \pi / 4}\right] .
$$

The complete operator sequence for the operator $U_{f_{4}}$ can then be written as

$$
U_{f_{4}}=\mathfrak{h}_{1}^{A}\left[\mathfrak{h}_{A}^{\prime \prime} h_{F}^{\prime} U_{\text {eff }}\right] \mathfrak{h}_{1}^{A} .
$$

\section{B. Deutsch-Jozsa operations}

The entire operation for the algorithm can be written in the form (see Fig. 1)

$$
U_{\mathrm{DJ}}=h_{1}^{F} U_{f_{n}} h_{1}^{F} \mathfrak{h}_{1}^{A},
$$

where time goes from right to left. Here we should mention that the operator $h_{1}$ given by (12) does not provide ideal Hadamard rotation, as $h_{1}^{2}$ is not equivalent to the identity operation, i.e.,

$$
\begin{gathered}
|0\rangle \stackrel{h_{1}}{\rightarrow} \frac{1}{\sqrt{2}}(|0\rangle+|1\rangle) \stackrel{h_{1}}{\rightarrow}|1\rangle, \\
|1\rangle \stackrel{h_{1}}{\rightarrow}-\frac{1}{\sqrt{2}}(|0\rangle-|1\rangle) \stackrel{h_{1}}{\rightarrow}-|0\rangle
\end{gathered}
$$

for the photon and similarly for qubit states $|g\rangle$ and $\left|g^{\prime}\right\rangle$ of a single atom. So, in this case, at the end of the operation $U_{f_{n}}$ in Eq. (29), we obtain the state $\left[\left(|0\rangle_{ \pm}|1\rangle\right)_{F} / \sqrt{2}\right] \Pi_{j=1}^{N}\left[\left(\left|g_{j}\right\rangle^{n}\right.\right.$ $\left.\left.+\left|g_{j}^{\prime}\right\rangle\right)_{A} / \sqrt{2}\right]$ up to overall phase factors, which is equivalent to the state $\left|\psi_{2}\right\rangle$ (4). The final $h_{1}^{F}$ operation on this state yields the $|1\rangle_{F}\left(|0\rangle_{F}\right)$ state for a constant (balanced) function.

Note that the operation $U_{\mathrm{DJ}}$ requires a large number of single-qubit rotation operators. We here emphasize that it is sufficient to perform less operations even without completing all the steps in $U_{f_{3}}$ or in $U_{f_{4}}$ to serve the purpose of the Deutsch-Jozsa algorithm. For example, we consider the operation $U_{f_{3}}$ given by Eq. (23). Then the relevant operator $U_{\mathrm{DJ}}$ to implement the entire algorithm can be written in the following form using Eqs. (23) and (29):

$$
U_{\mathrm{DJ}}=h_{1}^{F}\left[\mathfrak{h}_{1}^{A}\left\{\mathfrak{h}_{A}^{\prime} h_{F}^{\prime} U_{\mathrm{eff}} \mathfrak{h _ { 1 }}\right] h_{1}^{F} \mathfrak{h}_{1}^{A},\right.
$$

where the square-bracketed term stands for the $U_{f_{3}}$ operation. Rearranging the above, we can write

$$
U_{\text {DJ }}=\left[\mathfrak{h}_{1}^{A} \mathfrak{h}_{A}^{\prime}\right]\left[h_{1}^{F} h_{F}^{\prime}\right]\left[U_{\text {eff }}\right]\left[h_{1}^{F}\right]\left[\mathfrak{h}_{1}^{A} \mathfrak{h}_{1}^{A}\right] .
$$

Because we measure only the polarization state of the photon at the detector (see Fig. 1), from the above expression it is clear that it is not really necessary to perform the operation $\mathfrak{h}_{1}^{A} \mathfrak{h}_{A}^{\prime}$ on the atomic qubit. Precisely, we write the required part of the above operator $U_{\mathrm{DJ}}$, which is sufficient to determine if the function is balanced: 


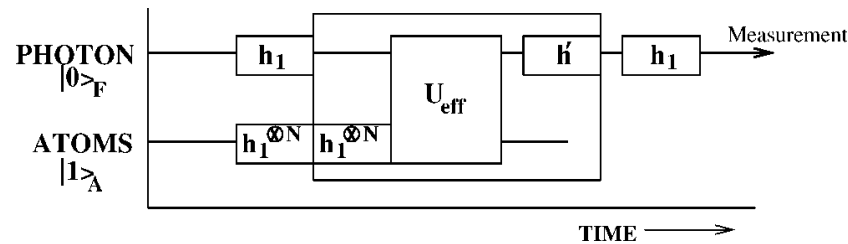

FIG. 4. Pulse sequence (33) is shown, which is sufficient to implement the Deutsch-Jozsa algorithm, using the $U_{f_{3}}$ or $U_{f_{4}}$ operator. The figure shows only the necessary part of the $U_{f_{n}}$ operation to complete the algorithm. One can measure the polarization state of the photonic qubit at the end of the process.

$$
U_{\mathrm{DJ}}^{\mathrm{eq}}=\left(h_{1}^{F} h_{F}^{\prime}\right) U_{\mathrm{eff}} h_{1}^{F}\left(\mathfrak{h}_{1}^{A} \mathfrak{h}_{1}^{A}\right),
$$

i.e., it is clear from Eq. (23) that for successful realization of the Deutsch-Jozsa algorithm, one need not perform the $U_{f_{3}}$ operation completely as one can ignore the atomic operations $\mathfrak{h}_{1}^{A} \mathfrak{h}_{A}^{\prime}$ at the end of the $U_{f_{3}}$ operation.

Interestingly, if we consider the operator $U_{f_{4}}$, the same equivalent operator sequence (33) is sufficient to perform the algorithm. We provide the corresponding operator sequence of $U_{\mathrm{DJ}}$ in the following:

$$
\begin{aligned}
U_{\text {DJ }} & =h_{1}^{F}\left[\mathfrak{h}_{1}^{A}\left\{\mathfrak{h}_{A}^{\prime \prime} h_{F}^{\prime} U_{\text {eff }} \mathfrak{h}_{1}^{A}\right] h_{1}^{F} \mathfrak{h}_{1}^{A}\right. \\
& =\left[\mathfrak{h}_{1}^{A} \mathfrak{h}_{A}^{\prime \prime}\right]\left[h_{1}^{F} h_{F}^{\prime}\right]\left[U_{\mathrm{eff}}\right]\left[h_{1}^{F}\right]\left[\mathfrak{h}_{1}^{A} \mathfrak{h}_{1}^{A}\right]=\left[\mathfrak{h}_{1}^{A} \mathfrak{h}_{A}^{\prime \prime}\right] U_{\mathrm{DJ}}^{\mathrm{eq}}
\end{aligned}
$$

In Fig. 4, we show the pulse sequence required to implement the Deutsch-Jozsa algorithm using the $U_{f_{3}}$ or $U_{f_{4}}$ operator. One first performs the operation $\mathfrak{h}_{1}^{A} \mathfrak{h}_{1}^{A}$ on the atomic ensemble and $h_{1}^{F}$ on the photon. As soon as these operations are over, the photon interacts with the atomic ensemble for a time $T=\pi / 2 N \lambda$ so that the two-qubit operation (11) occurs. Then one performs the final one-qubit operations for the photon. Thereby, the equivalent operation (33) for the algorithm is complete.

\section{CONCLUSIONS}

In conclusion, we have described a scheme to implement the Deutsch-Jozsa (DJ) algorithm. The scheme is based on a dispersive interaction of a macroscopic sample of $N$ atoms with a photon. Thus the ac Stark shift of the atomic ground levels leads to an effective Hamiltonian that has a form similar to the one used for quantum computation experiments with nuclear magnetic resonance systems. We properly choose different two-qubit unitary operators to perform the algorithm without actually producing CNOT or zero-CNOT (Z-CNOT) operations as in an ideal DJ algorithm. However for a specific choice of $N$ one can get CNOT, Z-CNOT, or quantum phase gate operations (as described in Appendix C). Moreover, the present scheme is experimentally more favorable as it can be implemented using an atomic ensemble interacting with a freely propagating photon. Note that single-photon sources are now becoming available [20]. Further, the chances of decoherence in the process are minimal as we deal with ground state atoms [21].

\section{APPENDIX A: DERIVATION OF THE HAMILTONIAN (8b)}

For simplicity, we consider two atoms in the ensemble. For a single photon interacting with these atoms, the wave function of the entire system can be spanned in terms of the relevant basis states as

$$
\begin{aligned}
|\psi(t)\rangle= & \left\{C_{g g 10}|g g 10\rangle+C_{g e 00}|g e 00\rangle+C_{e g 00}|e g 00\rangle\right\} \\
& +\left\{C_{g g^{\prime} 10}\left|g g^{\prime} 10\right\rangle+C_{e g^{\prime} 00}\left|e g^{\prime} 00\right\rangle\right\}+\left\{C_{g g^{\prime} 01}\left|g g^{\prime} 01\right\rangle\right. \\
& \left.+C_{g e^{\prime} 00}\left|g e^{\prime} 00\right\rangle\right\}+\left\{C_{g^{\prime} g 10}\left|g^{\prime} g 10\right\rangle+C_{g^{\prime} e 00}\left|g^{\prime} e 00\right\rangle\right\} \\
& +\left\{C_{g^{\prime} g 01}\left|g^{\prime} g 01\right\rangle+C_{e^{\prime} g 00}\left|e^{\prime} g 00\right\rangle\right\}+\left\{C_{g^{\prime} g^{\prime} 01}\left|g^{\prime} g^{\prime} 01\right\rangle\right. \\
& \left.+C_{g^{\prime} e^{\prime} 00}\left|g^{\prime} e^{\prime} 00\right\rangle+C_{e^{\prime} g^{\prime} 00}\left|e^{\prime} g^{\prime} 00\right\rangle\right\},
\end{aligned}
$$

where $|\alpha \beta n m\rangle$ refers to the state with atom 1 (2) in state $|\alpha\rangle(|\beta\rangle)$ and the mode $a_{+}\left(a_{-}\right)$in Fock state $|n\rangle(|m\rangle)$ and $C_{\alpha \beta n m}$ is the corresponding probability amplitude. Here we have grouped different subspaces in brackets. We use the interaction Hamiltonian (5) in the Schrödinger equation to derive the probability amplitude equations for all the basis states and use the adiabatic approximation $\Delta \gg g$. In this limit, as the levels $|e\rangle$ and $\left|e^{\prime}\right\rangle$ of both the atoms are never populated, the variation of the amplitudes $C_{g e 00}, C_{e g 00}$, $C_{g^{\prime} e^{\prime} 00}, C_{e^{\prime} g^{\prime} 00}, C_{e g^{\prime} 00}, C_{g e^{\prime} 00}, C_{g^{\prime} e 00}$, and $C_{e^{\prime} g 00}$ vanishes. After adiabatically eliminating these amplitudes in the remaining amplitude equations, we get the following:

$$
\begin{gathered}
i \dot{C}_{g g 10}=\frac{2|g|^{2}}{\Delta} C_{g g 10}, \quad i \dot{C}_{g^{\prime} g^{\prime} 01}=\frac{2|g|^{2}}{\Delta} C_{g^{\prime} g^{\prime} 01}, \\
i \dot{C}_{g g^{\prime} 10}=\frac{|g|^{2}}{\Delta} C_{g g^{\prime} 10}, \quad i \dot{C}_{g g^{\prime} 01}=\frac{|g|^{2}}{\Delta} C_{g g^{\prime} 01}, \\
i \dot{C}_{g^{\prime} g 10}=\frac{|g|^{2}}{\Delta} C_{g^{\prime} g 10}, \quad i \dot{C}_{g^{\prime} g 01}=\frac{|g|^{2}}{\Delta} C_{g^{\prime} g 01} .
\end{gathered}
$$

We thus can write an effective Hamiltonian corresponding to the above equations as

$$
\begin{aligned}
H_{\mathrm{eff}}= & \frac{2 \hbar|g|^{2}}{\Delta}\left[|g g 10\rangle\left\langle g g 10|+| g^{\prime} g^{\prime} 01\right\rangle\left\langle g^{\prime} g^{\prime} 01\right|\right] \\
& +\frac{\hbar|g|^{2}}{\Delta}\left[\left|g g^{\prime} 10\right\rangle\left\langle g g^{\prime} 10|+| g g^{\prime} 01\right\rangle\left\langle g g^{\prime} 01\right|\right. \\
& \left.+\left|g^{\prime} g 10\right\rangle\left\langle g^{\prime} g 10|+| g^{\prime} g 01\right\rangle\left\langle g^{\prime} g 01\right|\right],
\end{aligned}
$$

After a little algebra, we can rewrite the above Hamiltonian in the following form:

$$
\begin{aligned}
\frac{H_{\mathrm{eff}}}{\hbar}= & \lambda\left[|g 10\rangle\left\langle g 10|+| g^{\prime} 01\right\rangle\left\langle g^{\prime} 01\right|\right]_{1}\left[|g\rangle\left\langle g|+| g^{\prime}\right\rangle\left\langle g^{\prime}\right|\right]_{2} \\
& +\lambda\left[|g\rangle\left\langle g|+| g^{\prime}\right\rangle\left\langle g^{\prime}\right|\right]_{1}\left[|g 10\rangle\left\langle g 10|+| g^{\prime} 01\right\rangle\left\langle g^{\prime} 01\right|\right]_{2},
\end{aligned}
$$

where the subscript $j$ refers to the $j$ th atom and $\lambda=|g|^{2} / \Delta$. As the levels $|e\rangle$ and $\left|e^{\prime}\right\rangle$ are never populated in the large detuning limit, we can approximate the terms $|g\rangle\left\langle g|+| g^{\prime}\right\rangle\left\langle g^{\prime}\right|$ to be a unit operator which does not contribute to the evolution of the system. Thus the Hamiltonian now reads as 


$$
H_{\text {eff }}=\hbar \lambda \sum_{j=1}^{2}\left[|g\rangle_{j}\langle g \| 1,0\rangle\left\langle 1,0|+| g^{\prime}\right\rangle_{j}\left\langle g^{\prime}|| 0,1\right\rangle\langle 0,1|\right],
$$

which is the same as Eq. (8b) for $N=2$.

\section{APPENDIX B: EQUATIONS USED IN NUMERICAL SIMULATIONS}

Let us consider the initial state of the atom-photon system to be $\left|1_{F}\right\rangle\left|0_{A}\right\rangle=\prod_{j=1}^{N}\left|g_{j}\right\rangle|1,0\rangle$. Then the total wave function of the system can be expanded in terms of the possible basis states as

$$
|\psi(t)\rangle=c_{0} \prod_{j=1}^{N}\left|g_{j}\right\rangle|1,0\rangle+\sum_{l=1}^{N} c_{l} \prod_{j \neq l}\left|g_{j}\right\rangle\left|e_{l}\right\rangle|0,0\rangle .
$$

Then using the Hamiltonian (5), we find the following amplitude equations:

$$
\begin{gathered}
\dot{c}_{0}=g^{*} d, \quad d=\sum_{l=1}^{N} c_{l}, \\
\dot{d}=i \Delta d-g N c_{0} .
\end{gathered}
$$

From the above equations, one can find out the analytical solution for $c_{0}$ as

$$
c_{0}(t)=e^{i \Delta t / 2}\left\{\frac{-i \Delta}{p} \sin \left(\frac{p t}{2}\right)+\cos \left(\frac{p t}{2}\right)\right\},
$$

where $p=\sqrt{\Delta^{2}+4 N|g|^{2}}$.

\section{APPENDIX C: WORKING WITH A SPECIFIC CHOICE OF $N$}

If one puts the constraint $N=8 m+1, m=0,1,2, \ldots$, on the number of atoms in the sample, then the evolution (21) can be written as

$$
\mathfrak{h}_{A}^{\prime}|0\rangle_{A}=e^{i \pi / 4}|0\rangle_{A}, \quad \mathfrak{h}_{A}^{\prime}|1\rangle_{A}=e^{-i \pi / 4}|1\rangle_{A} .
$$

Thus the operation $Q_{1}$ represents a phase gate given by

$$
Q_{1}=\operatorname{diag}[i,-i,-i,-i]
$$

in the $\left(\left|0_{F} 0_{A}\right\rangle,\left|0_{F} 1_{A}\right\rangle,\left|1_{F} 0_{A}\right\rangle,\left|1_{F} 1_{A}\right\rangle\right)$ basis. Then the operator $U_{f_{3}}$ can be identified as a CNOT gate

$$
U_{f_{3}}=i\left(\begin{array}{cccc}
1 & 0 & 0 & 0 \\
0 & -1 & 0 & 0 \\
0 & 0 & 0 & 1 \\
0 & 0 & -1 & 0
\end{array}\right)
$$

in the same basis as is derived by applying Hadamard rotations before and after the phase gate operation $Q_{1}$ [Eq. (C2)]. In a similar fashion, for the same choice of $N$, the operator $Q_{2}$ represents the following phase gate operation:

$$
Q_{2}=\operatorname{diag}[1,1,-1,1]
$$

in the $\left(\left|0_{F} 0_{A}\right\rangle,\left|0_{F} 1_{A}\right\rangle,\left|1_{F} 0_{A}\right\rangle,\left|1_{F} 1_{A}\right\rangle\right)$ basis. Thus the $U_{f_{4}}$ represents a Z-CNOT gate given by

$$
U_{f_{4}}=\left(\begin{array}{cccc}
0 & -1 & 0 & 0 \\
1 & 0 & 0 & 0 \\
0 & 0 & -1 & 0 \\
0 & 0 & 0 & 1
\end{array}\right)
$$

in the same basis. Note that this gate inverts the state of the atomic qubit only when the photonic qubit is in the $|0\rangle_{F}$ state.
[1] D. Deutsch, Proc. R. Soc. London, Ser. A 400, 97 (1985); D. Deutsch and R. Jozsa, ibid. 493, 553 (1992).

[2] M. A. Nielsen and I. L. Chuang, Quantum Computation and Quantum Information (Cambridge University Press, Cambridge, U.K., 2002), p. 34.

[3] L. K. Grover, Phys. Rev. Lett. 79, 325 (1997); 79, 4709 (1997); 80, 4329 (1998).

[4] I. L. Chuang, I. M. K. Vandersypen, X. Zhou, D. W. Leung, and S. Lloyd, Nature (London) 393, 143 (1998); T. F. Jones and M. Mosca, J. Chem. Phys. 109, 1648 (1998).

[5] I. L. Chuang, N. Gershenfeld, and M. Kubinec, Phys. Rev. Lett. 80, 3408 (1998).

[6] K. Dorai, Arvind, and A. Kumar, Phys. Rev. A 61, 042306 (2000).

[7] R. Das and A. Kumar, Phys. Rev. A 68, 032304 (2003).

[8] S. Guide, M. Riebe, G. P. T. Lancaster, C. Becher, J. Eschner, H. Häffner, F. Schmidt-Kaler, I. L. Chuang, and R. Blatt, Nature (London) 421, 48 (2003).

[9] M. Feng, Phys. Rev. A 63, 052308 (2001).

[10] F. Yamaguchi, P. Milman, M. Brune, J. M. Raimond, and S. Haroche, Phys. Rev. A 66, 010302 (2002).

[11] M. A. Nielsen and I. L. Chuang, Quantum Computation and
Quantum Information (Ref. [2]), p. 337.

[12] A. Kuzmich, N. P. Bigelow, and L. Mandel, Europhys. Lett. 42, 481 (1998); A. Kuzmich, L. Mandel, J. Janis, Y. E. Young, R. Ejnisman, and N. P. Bigelow, Phys. Rev. A 60, 2346 (1999); A. Kuzmich, L. Mandel, and N. P. Bigelow, Phys. Rev. Lett. 85, 1594 (2000).

[13] S. Massar and E. S. Polzik, Phys. Rev. Lett. 91, 060401 (2003); G. S. Agarwal, P. Lougovski, and H. Walther (unpublished).

[14] L.-M. Duan, J. I. Cirac, P. Zoller, and E. S. Polzik, Phys. Rev. Lett. 85, 5643 (2000).

[15] I. E. Protsenko, G. Reymond, N. Schlosser, and P. Grangier, Phys. Rev. A 66, 062306 (2002).

[16] Y. Takahashi, K. Honda, N. Tanaka, K. Toyoda, K. Ishikawa, and T. Yabuzaki, Phys. Rev. A 60, 4974 (1999).

[17] A. Kuzmich and E. S. Polzik, Phys. Rev. Lett. 85, 5639 (2000).

[18] M A. Nielsen and I L. Chuang, Quantum Computation and Quantum Information (Ref. [2]), p. 287.

[19] T. B. Pittman, B. C. Jacobs, and J. D. Franson, Phys. Rev. Lett. 88, 257902 (2002); J. D. Franson, M. M. Donegan, M. J. Fitch, B. C. Jacobs, and T. B. Pittman, ibid. 89, 137901 
(2002)

[20] A. Kuhn, M. Hennrich, T. Bondo, and G. Rempe, Appl. Phys. B: Lasers Opt. 69, 373 (1999); A. Kuhn, M. Hennrich, and G. Rempe, Phys. Rev. Lett. 89, 067901 (2002).

[21] The implementation of the present proposal using trapped atoms in a cavity [J. McKeever, J. R. Buck, A. D. Boozer, A.
Kuzmich, H.-C. Nägerl, D. M. Stamper-Kurn, and H. J. Kimble, Phys. Rev. Lett. 90, 133602 (2003); J. McKeever, J. R. Buck, A. D. Boozer, and H. J. Kimble, e-print quant-ph/ 0403121] will be possible if one can fix the time of interaction such that $\lambda N T=\pi / 2$. 